\title{
Diseño y construcción de un Colector Solar de Tipo Concentrador Parabólico Compuesto (CPC) para pruebas de calentamiento de aire
}

\section{Design and construction of a Compound Parabolic Concentrator (CPC) Solar collector for air heating testing}

\author{
SORIA-MEJÍA, Sandra Paloma†, AMBROSIO-JUAREZ, José Emmanuel, TOLEDO-MANUEL, Iván \\ y SABÁS-SEGURA, José* \\ Instituto Tecnológico Superior de Abasolo-Ingeniería en Energías Renovables \\ ID 1er Autor: Sandra Paloma, Soria-Mejía / ORC ID: 0000-0002-0664-0688 \\ ID $1^{\mathrm{er}}$ Coautor: José Emmanuel, Ambrosio-Juarez / ORC ID: 0000-0002-4741-9269, CVU CONACYT ID: 563918 \\ ID $2^{\text {do }}$ Coautor: Iván, Toledo-Manuel / ORC ID: 0000-0001-9498-5506, CVU CONACYT ID: 477272 \\ ID $3^{\text {er }}$ Coautor: José, Sabás-Segura / ORC ID: 0000-0003-4579-0192, CVU CONACYT ID: 891182
}

DOI: 10.35429/JCPE.2019.19.6.22.30

Recibido 09 Abril, 2019; Aceptado 28 Junio, 2019

\section{Resumen}

En este trabajo se presenta una propuesta de diseño y la evaluación teórica y experimental de un colector solar de tipo concentrador parabólico compuesto (CPC) con absorbedor tubular para pruebas de calentamiento de aire con rendimientos térmicos ideales para aplicaciones como el secado y sistemas de acondicionamiento de aire. El colector consiste en cuatro concentradores formados de lámina galvanizada que hacen la función de reflejar la luz solar hacia los tubos absorbedores, con una relación de concentración de 2.5 y un semiángulo de aceptación de $23.5^{\circ}$, con el objetivo de no requerir de un sistema de seguimiento solar. El absorbedor es un tubo de cobre donde el aire circula. Con esta propuesta del colector se obtuvo una eficiencia teórica de $25 \%$, lo que representa una tercia parte de ganancia de energía comparándolo con un CPC comercial. Esta eficiencia se obtuvo de acuerdo a lo materiales y dimensiones implementados. El sistema es técnicamente factible de utilizarse para procesos de baja temperatura, con calidad promedio de $60^{\circ} \mathrm{C}$, con lo que se puede producir ahorros energéticos significativos y reduciendo la cantidad de emisiones de $\mathrm{CO} 2$, favoreciendo al medio ambiente.

Colector solar, CCP, Calentamiento de aire

\begin{abstract}
This paper presents a design proposal and the theoretical and experimental evaluation of a solar collector of parabolic concentrator type (CPC) with tubular absorber for air heating tests with ideal thermal performances for applications such as drying and conditioning systems. air. The collector consists of four concentrators formed of galvanized sheet that make the function of reflecting the sunlight towards the absorber tubes, with a concentration ratio of 2.5 and an acceptance half-angle of $23.5^{\circ}$, with the objective of not requiring a system of solar tracking The absorber is a copper tube where the air circulates. With this proposal from the collector, a theoretical efficiency of $25 \%$ was obtained, which represents a third part of energy gain compared to a commercial CPC. This efficiency was obtained according to the materials and dimensions implemented. The system is technically feasible to be used for low temperature processes, with average quality of 60 ${ }^{\circ} \mathrm{C}$, which can produce significant energy savings and reducing the amount of $\mathrm{CO} 2$ emissions, favoring the environment.
\end{abstract}

Solar collector, CCP, Air heating

Citación: SORIA-MEJÍA, Sandra Paloma, AMBROSIO-JUAREZ, José Emmanuel, TOLEDO-MANUEL, Iván y SABÁSSEGURA, José. Diseño y construcción de un Colector Solar de Tipo Concentrador Parabólico Compuesto (CPC) para pruebas de calentamiento de aire. Revista de Energía Química y Física. 2019. 6-19: 22-30

\footnotetext{
* Correspondencia al Autor (Correo electrónico: jose.sabas@ @ecabasolo.edu.mx)

$\dagger$ Investigador contribuyendo como primer Autor
} 


\section{Introducción}

El crecimiento demográfico y su constante desarrollo generan un desequilibrio ecológico en el planeta, generando fuentes de contaminación que conllevan el deterioro de los recursos naturales y el calentamiento global incontrolado. México presenta una fuerte dependencia de los combustibles fósiles para la generación de energía eléctrica, presentando la tasa de crecimiento anual de consumo de electricidad más alta entre los países de EUA y Canadá con $3.4 \%$, por efecto de la progresiva demanda de recursos energéticos necesarios para su expansión económica (SENER, 2015).

El uso de las fuentes de energías renovables para la producción de energía eléctrica y calor en los distintos sectores reduciría en gran medida los problemas económicos y medio ambientales en nuestro país. La tecnología que apunta a tener un mayor auge es la energía solar. En México se cuenta con una excelente radiación solar, disponiendo en promedio anual de 4.5 a $5.6 \mathrm{kWh} / \mathrm{m} 2$ de radiación global media diaria (Herrera, 2018).

El fin de este proyecto es utilizar la energía solar disponible mediante el diseño y la fabricación de un colector solar de tipo concentrador parabólico compuesto (CPC) como generador de aire caliente como un instrumento de pruebas para cualquier aplicación que requiera ser utilizado en hora solar punta a una temperatura inferior a $60^{\circ}$ y que además pueda ser utilizado como una herramienta para el ahorro de energía. Con este trabajo se quiere fomentar una mayor investigación en el campo de la energía solar térmica, para aplicaciones en condiciones controladas para procesos de secado o desinfección a temperaturas que no superen los $70^{\circ}$.

Los CPC logran operar entre 30 a $200{ }^{\circ} \mathrm{C}$ (UNAM, 2018), así pues, en el presente trabajo se profundiza en el diseño, construcción y análisis del CPC como dispositivo captador de radiación solar haciendo fluir el aire a través de los tubos absorbedores para algún proceso. El CPC se diseña empleando materiales que permiten la captación y absorción de la radiación solar recibida en el lugar, construyendo un colector solar con cuatro reflectores de lámina de acero galvanizada para formar los concentradores y redirigir la radiación solar desde la abertura hasta los receptores.
Se agrega un receptor por cada concentrador, los cuales son encargados de interceptar la energía solar y transformarla en energía térmica, empleando tubos de cobre por ser un buen conductor térmico. Su estructura es realizada a base de materiales metálicos para una mejor rigidez y duración.

\section{Diseño del Colector Parabólico Compuesto (CPC)}

El factor más importante al diseñar este tipo de colector es la relación de concentración, que, para un sistema lineal, viene dada por la ecuación 1. (Duffie \& Beckman, 2013)

$\mathrm{C}=\frac{\mathrm{A}_{\mathrm{a}}}{\mathrm{A}_{\mathrm{r}}}=\frac{1}{\sin \theta_{\mathrm{a}}}$

Para colectores CPC estacionarios, el ángulo de aceptación mínimo deberá ser igual a $47^{\circ}$, este ángulo será capaz de cubrir la declinación del sol desde el solsticio de verano hasta el solsticio de invierno $\left(2 \times 23.5^{\circ}\right)$. (López, 2011)

Un CPC con absorbedor tubular se diseña en base a la relación de concentración que se requiere y al diámetro exterior del tubo absorbedor. Para la construcción de la parábola y la involuta del CPC se toma como referencia la figura 1.

Donde $\mathrm{S}$ es un punto genérico en el reflector, el segmento RS es la tangente al tubo absorbedor, $\mathrm{O}$ es el centro del tubo, $\mathrm{r}$ es el radio del tubo, $\mathrm{AB}$ es la parte de la involuta y $\mathrm{BC}$ es la parábola de la curva compuesta.

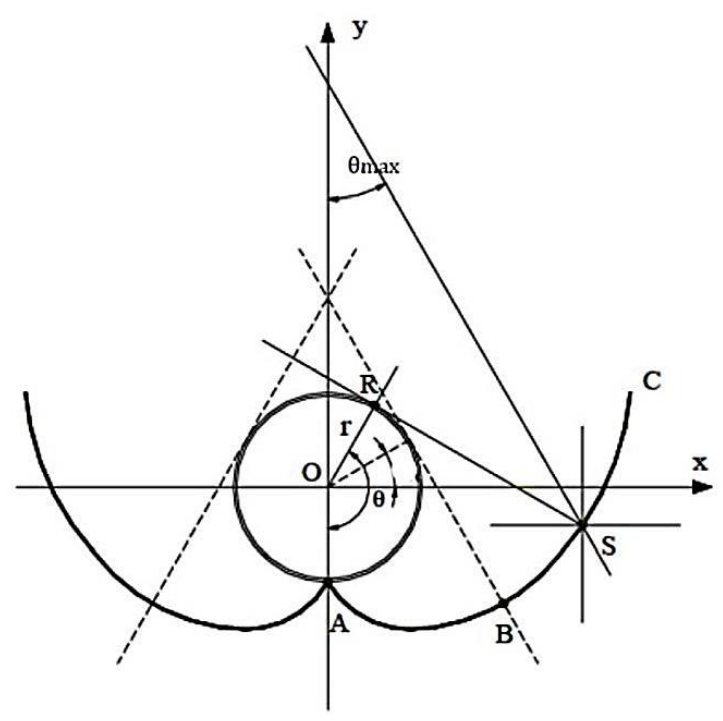

Figura 1 Descripción del CPC para un receptor tubular Fuente: Blanco Gálvez et al , 2006

SORIA-MEJÍA, Sandra Paloma, AMBROSIO-JUAREZ, José Emmanuel, TOLEDO-MANUEL, Iván y SABÁS-SEGURA, José. Diseño y construcción de un Colector Solar de Tipo Concentrador Parabólico Compuesto (CPC) para pruebas de calentamiento de aire. Revista de Energía Química y Física. 2019 
La ecuación 2, define el rango de la curva de la involuta donde $\theta$ está dado en radianes:

$0 \leq \theta \leq\left[\left(\frac{\pi}{2}\right)+\theta_{\mathrm{a}}\right]$

Las siguientes ecuaciones definen las coordenadas $\mathrm{x}$, y para obtener la involuta de la curva compuesta:

$\mathrm{x}=\mathrm{r}(\sin \theta-\theta \cos \theta)$

$y=r(-\cos \theta-\theta \sin \theta)$

Para la parte de la parábola en la curva compuesta el rango para el valor del ángulo de diseño $\theta$ va de acuerdo a la siguiente ecuación:

$\left[\left(\frac{\pi}{2}\right)+\theta_{\mathrm{a}}\right] \leq \theta \leq\left[\left(\frac{3 \pi}{2}\right)-\theta_{\mathrm{a}}\right]$

Donde las ecuaciones para las coordenadas $\mathrm{x}$, y de la parábola son:

$\mathrm{x}=\mathrm{r}\left[\sin \theta-\left\{\frac{\frac{\pi}{2}+\theta_{\mathrm{a}}+\theta-\cos \left(\theta-\theta_{\mathrm{a}}\right)}{\left(1+\sin \left(\theta-\theta_{\mathrm{a}}\right)\right)}\right\}(\cos \theta)\right]$

$y=r\left[-\cos \theta-\left\{\frac{\frac{\pi}{2}+\theta_{a}+\theta-\cos \left(\theta-\theta_{a}\right)}{\left(1+\sin \left(\theta-\theta_{a}\right)\right)}\right\}(\operatorname{sen} \theta)\right]$

Las ecuaciones anteriores son programadas en el software Matlab para obtener la geometría del CPC, teniendo como resultado la figura 2 , en donde el semiángulo de aceptación $\theta \mathrm{a}$ es igual a $23.5^{\circ}$ y el diámetro del tubo absorbedor es de $0.0142875 \mathrm{~m}$, para un índice de concentración de 2.5 , el cual se encuentra dentro del rango válido para este tipo de colector.

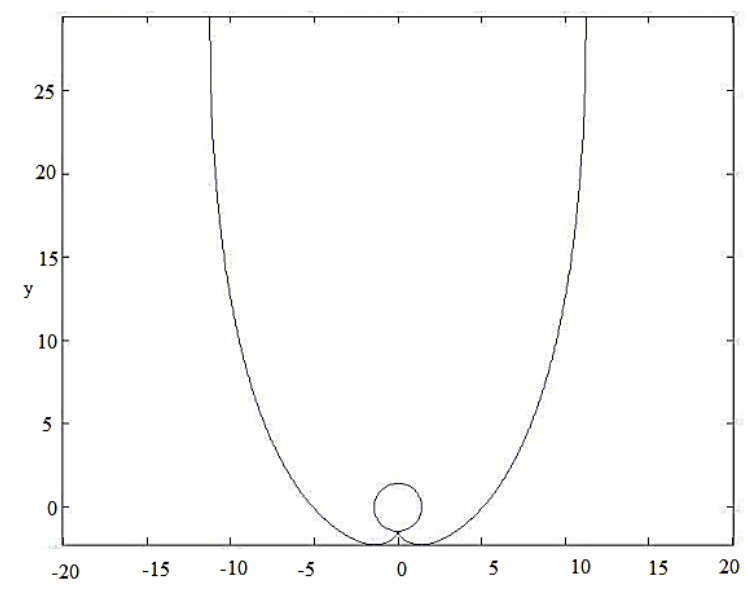

Figura 2 Geometría del Colector cilíndrico parabólico
A continuación, se simula el comportamiento óptico del CPC en el software Tonatiuh, en donde se realiza el trazado de rayos desde una fuente solar supuesta hacia el CPC, y así observar la reflexión y recepción de los rayos solares. En la figura 3, se puede observar los resultados obtenidos de la simulación, donde se percata que los rayos solares entran en el área de abertura del colector, luego son reflejados por la lámina para después ser captados y absorbidos por el tubo receptor.

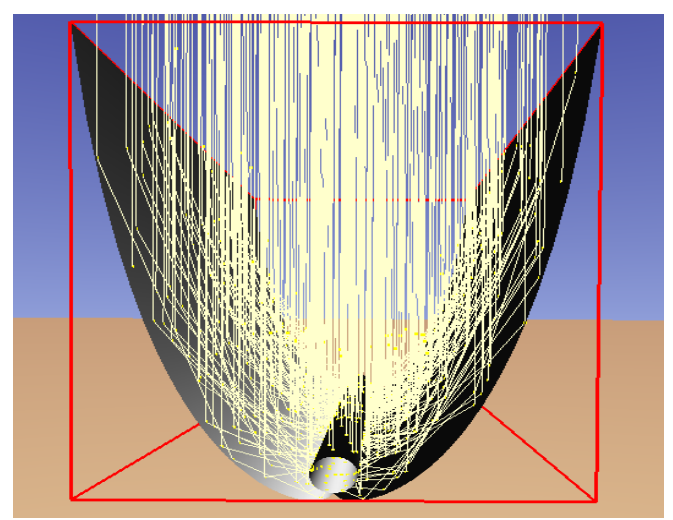

Figura 3 Comportamiento óptico del CPC simulado en Tonatiuh.

\section{Análisis Teórico del Rendimiento Térmico del CPC}

El cálculo del rendimiento de los colectores de concentración sigue los mismos lineamientos generales que para los colectores de placa plana (Duffie \& Beckman, 2013), usando la siguiente ecuación:

$\eta=\frac{\mathrm{Q}_{\mathrm{u}}}{\mathrm{S} \cdot \mathrm{A}_{\mathrm{c}}}$

Para determinar el calor útil $(\mathrm{Qu})$ se implementa la ecuación 9:

$Q_{u}=F_{R} \cdot A_{a}\left[S-\frac{A_{r}}{A_{a}} U_{L}\left(T_{s}-T_{a}\right)\right]$

Donde la energía que entra al sistema se deriva de la radiación solar absorbida por la tubería receptora del colector (S), la cual se estima para cielo despejado a partir de la siguiente ecuación condicionada por la geometría del colector:

$\mathrm{S}=\mathrm{A}_{\mathrm{C}}\left(\mathrm{G}_{\mathrm{b}} \cdot \tau_{\mathrm{c}, \mathrm{b}} \cdot \tau_{\mathrm{CPC}, \mathrm{b}} \cdot \alpha_{\mathrm{b}}+\mathrm{G}_{\mathrm{d}} \cdot \tau_{\mathrm{c}, \mathrm{d}} \cdot\right.$

$\left.\tau_{\mathrm{CPC}, \mathrm{d}} \cdot \alpha_{\mathrm{d}}+\mathrm{G}_{\mathrm{g}} \cdot \tau_{\mathrm{c}, \mathrm{g}} \cdot \tau_{\mathrm{CPC}, \mathrm{g}} \cdot \alpha_{\mathrm{g}}\right)$ 
Luego es necesario estimar la contribución de la radiación directa ( $\mathrm{G}$ subíndice $\mathrm{b}$, por beam radiation), la radiación difusa ( $\mathrm{G}$ subíndice d, por diffuse radiation) y la radiación reflejada por el suelo (G subíndice g, por groundreflected radiation), donde se consideran los criterios de las ecuaciones 11 y 12 :

$\mathrm{G}_{\mathrm{b}}=\mathrm{F} \cdot \mathrm{G}_{\mathrm{bn}} \cdot \cos \theta$

$G_{d}= \begin{cases}\frac{G_{d}}{C} & \text { si }\left(\beta+\theta_{a}\right)<90^{\circ} \\ \frac{G_{d}}{2}\left(\frac{1}{C}-\cos \beta\right) & \text { si }\left(\beta+\theta_{a}\right)>90^{\circ}\end{cases}$

$\mathrm{G}_{\mathrm{g}}= \begin{cases}0 & \text { si }\left(\beta+\theta_{\mathrm{a}}\right)<90^{\circ} \\ \frac{\mathrm{G}_{\mathrm{g}}}{2}\left(\frac{1}{\mathrm{c}}-\cos \beta\right) & \text { si }\left(\beta+\theta_{\mathrm{a}}\right)>90^{\circ}\end{cases}$

De la ecuaciones 12 y 13, según la condición $\beta+\Theta a$ para este caso y sabiendo que el ángulo de inclinación respecto a la superficie horizontal $(\beta)$ de este colector es $21^{\circ}$ y el semiángulo de aceptación $\Theta a$ es igual a $23.5^{\circ}$, se tiene $\beta+\Theta a=44.5^{\circ}$, que será menor que $90^{\circ}$, lo que conlleva a resolver la ecuación 12 para radiación difusa.

$\mathrm{G}_{\mathrm{d}}=\frac{\mathrm{G}_{\mathrm{d}}}{\mathrm{C}}$

En cuanto a la radiación reflejada por el suelo para este diseño se despreciara por ser prácticamente nula, cumpliéndose la primera condición:

$\mathrm{G}_{\mathrm{g}}=0$

Con los puntos anteriores determinados, la ecuación 10 resulta de la siguiente manera:

$\mathrm{S}=\mathrm{A}_{\mathrm{C}}\left(\mathrm{G}_{\mathrm{b}} \cdot \tau_{\mathrm{c}, \mathrm{b}} \cdot \tau_{\mathrm{CPC}, \mathrm{b}} \cdot \alpha_{\mathrm{b}}+\mathrm{G}_{\mathrm{d}} \cdot \tau_{\mathrm{c}, \mathrm{d}} \cdot\right.$

$\left.\tau_{\mathrm{CPC}, \mathrm{d}} \cdot \alpha_{\mathrm{d}}\right)$

El colector propuesto no posee cobertura, ni en la estructura ni en los absorbedores por lo tanto:

$\tau_{c, b}=\tau_{c, d}=\tau_{c}=1$

Para encontrar el valor de la transmisividad efectiva del CPC $\left(\tau_{\mathrm{CPC}}\right)$, se toma en cuenta la fracción de la radiación que pasa a través de la abertura del colector que se concentra en el receptor donde la reflectividad especular de las paredes de los concentradores ( $\rho r)$ y del número promedio de reflexiones (nr), esta transmitancia se expresa como: (Duffie \& Beckman, 2013)
$\tau_{\mathrm{CPC}, \mathrm{b}}=\tau_{\mathrm{CPC}, \mathrm{d}}=\tau_{\mathrm{CPC}}=\rho_{\mathrm{r}}^{\mathrm{n}_{\mathrm{r}}}$

$\mathrm{n}_{\mathrm{r}}=1-\frac{1}{\mathrm{C}_{\mathrm{m}}}$

Se debe conocer la absortancia de la superficie receptora de cobre ( $\alpha$ r) para la radiación, que será el mismo valor para la radiación directa $(\alpha b)$ y la radiación difusa $(\alpha d)$. Entonces de acuerdo con (Duffie \& Beckman, 2013), la ecuación 16 se reduce a la ecuación 20 , donde la radiación solar total $(\mathrm{G})$ es la suma de las radiaciones directa $(\mathrm{Gb})$ y difusa $(\mathrm{Gd})$ :

$\mathrm{S}=\mathrm{A}_{\mathrm{c}} \cdot \mathrm{G}\left(\tau_{\mathrm{r}} \cdot \tau_{\mathrm{CPC}} \cdot \alpha_{\mathrm{r}}\right)$

$\mathrm{G}=\mathrm{G}_{\mathrm{b}}+\mathrm{G}_{\mathrm{d}}$

(Hottel, 1976) ha presentado un método para estimar la radiación transmitida a través de atmósferas claras que toma en cuenta el ángulo cenital y la altitud para una atmósfera estándar y para cuatro tipos de clima (Duffie \& Beckman, 2013), el cual es utilizado para hallar la radiación directa y difusa mediante las siguientes ecuaciones:

$\mathrm{G}_{\mathrm{b}}=\mathrm{G}_{\mathrm{o}} \cdot \tau_{\mathrm{b}}$

$\mathrm{G}_{\mathrm{d}}=\mathrm{G}_{\mathrm{o}} \cdot \tau_{d}$

Donde la irradiancia extraterrestre se puede conocer a partir de la ecuación 24:

$\mathrm{G}_{\mathrm{o}}=1367\left(1+0.033 \cdot \cos \frac{360 \cdot \mathrm{N}}{365}\right)$

Para determinar las transmitancias atmosféricas para la radiación directa $\left(\tau_{\mathrm{b}}\right)$ y difusa $\left(\tau_{\mathrm{d}}\right)$, se emplean las ecuaciones descritas a continuación:

$\tau_{\mathrm{b}}=\mathrm{a}_{0}+\mathrm{a}_{1} \exp \left(-\frac{\mathrm{k}}{\cos \theta_{\mathrm{z}}}\right)$

$\tau_{\mathrm{d}}=0.271-0.294 \cdot \tau_{\mathrm{b}}$

Donde los valores $a 0$, al y $k$ para la atmósfera estándar se calculan a partir de las ecuaciones siguientes para altitudes inferiores a $2.5 \mathrm{~km}$, así como también se requiere conocer el coseno del ángulo cenital $(\Theta z)$ que viene dado por la ecuación 30: (Hottel, 1976)

$\mathrm{a}_{0}=0.4237-0.0082(6-\mathrm{A})^{2}$

$\mathrm{a}_{1}=0.5055-0.00595(6.5-\mathrm{A})^{2}$

$\mathrm{k}=0.2711-0.01858(2.5-\mathrm{A})^{2}$

$\cos \theta_{\mathrm{z}}=\cos \Phi \cdot \cos \delta \cdot \cos \omega+\sin \Phi \cdot \sin \delta$

SORIA-MEJÍA, Sandra Paloma, AMBROSIO-JUAREZ, José Emmanuel, TOLEDO-MANUEL, Iván y SABÁS-SEGURA, José. Diseño y construcción de un Colector Solar de Tipo Concentrador Parabólico Compuesto (CPC) para pruebas de calentamiento de aire. Revista de Energía Química y Física. 2019 
En la que el ángulo de declinación solar

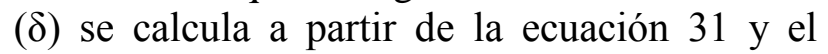
ángulo horario $(\omega)$ de la ecuación 32 , la cual solicita el número del tiempo solar (Tsun) conociéndolo por medio de la ecuación 33, que conlleva a pedir el número del tiempo del reloj actual (Tclock) y a resolver la Ecuación del Tiempo (ET). (Duffie \& Beckman, 2013)

$$
\begin{aligned}
& \delta=23.45 \sin \left[\frac{360}{365}(284+\mathrm{N})\right] \\
& \omega=\left(\mathrm{T}_{\text {sun }}-12\right) \cdot 15 \\
& \mathrm{~T}_{\text {sun }}=\mathrm{T}_{\text {clock }}+\mathrm{ET} \pm 4\left(\mathrm{~L}_{\text {std }}-\mathrm{L}_{\text {loc }}\right) \\
& \mathrm{ET}=9.87 \sin (2 \mathrm{~B})-7.53 \cos \mathrm{B}-1.5 \sin \mathrm{B} \\
& \mathrm{B}=(\mathrm{N}-81) \frac{360}{365}
\end{aligned}
$$

Ahora bien, se procede a calcular la energía que sale del sistema y esta viene dada por las pérdidas térmicas del receptor, teniendo en cuenta la convección y la radiación de la superficie, así como también la conducción a través de la estructura de soporte, las cuales deben estimarse generalmente en términos del coeficiente de pérdida UL, que se basa en el área del receptor y está dado por:

$$
\mathrm{U}_{\mathrm{L}}=\frac{\mathrm{Q}_{\text {loss }}}{\pi \cdot \mathrm{e} \cdot \mathrm{L}\left(\mathrm{T}_{\mathrm{r}}-\mathrm{T}_{\mathrm{a}}\right)}
$$

La pérdida de calor del receptor (Qloss), está dada por lo siguiente:

$$
\mathrm{Q}_{\text {loss }}=\frac{\pi \mathrm{D}_{\mathrm{e}} \mathrm{L} \sigma\left(\mathrm{T}_{\mathrm{r}}^{4}-\mathrm{T}_{\mathrm{i}}^{4}\right)}{\frac{1}{\varepsilon_{\mathrm{r}}}+\frac{1-\varepsilon_{\mathrm{r}}}{\varepsilon_{\mathrm{r}}}\left(\frac{\mathrm{D}_{\mathrm{e}}}{\mathrm{D}_{\mathrm{i}}}\right)}
$$

Para encontrar el factor de eliminación de calor del colector (FR) se despeja de la ecuación 38, encontrando el factor de flujo del colector (F"') de la ecuación 40 y el factor de eficiencia ( $F^{\prime}$ ) de la ecuación 41: (Duffie \& Beckman, 2013)

$$
\begin{aligned}
& F^{\prime \prime}=\frac{F_{R}}{F^{\prime}} \\
& F^{\prime \prime}=\frac{\dot{m} \cdot C_{p}}{A_{r} \cdot U_{L} \cdot F^{\prime}}\left[1-\exp \left(-\frac{A_{r} \cdot U_{L} \cdot F^{\prime}}{\dot{m} \cdot C_{p}}\right)\right] \\
& F^{\prime}=\frac{U_{0}}{U_{L}}
\end{aligned}
$$

Donde el flujo másico m se calcula primeramente para la ecuación 39 mediante la densidad del fluido, la velocidad promedio y el área de la sección transversal normal a la dirección del flujo (Ar).

$\dot{\mathrm{m}}=\rho \cdot \mathrm{V} \cdot \operatorname{Ar}$

El coeficiente global de transferencia de calor basado en el diámetro del tubo receptor externo (Uo) entre el entorno y el fluido se calcula como se muestra en la ecuación 42:

$$
\mathrm{U}_{\mathrm{o}}=\left(\frac{1}{\mathrm{U}_{\mathrm{L}}}+\frac{\mathrm{D}_{\mathrm{e}}}{\mathrm{h}_{\mathrm{fi}} \cdot \mathrm{D}_{\mathrm{i}}}+\frac{\mathrm{D}_{\mathrm{e}} \cdot \ln \left(\mathrm{D}_{\mathrm{e}} / \mathrm{D}_{\mathrm{i}}\right)}{2 \cdot \mathrm{k}_{\mathrm{r}}}\right)^{-1}
$$

En el cual el coeficiente de transferencia de calor del fluido de trabajo (hfi) se basa en el diámetro interior del tubo receptor y para estimarlo se despeja de la siguiente ecuación:

$\mathrm{Nu}=\frac{\mathrm{h}_{\mathrm{fi}} \cdot \mathrm{D}_{\mathrm{i}}}{\mathrm{k}_{\text {aire }}}$

Donde el número de Nusselt se encuentra tomando en cuenta las siguientes condiciones: (Duffie \& Beckman, 2013).

$$
\mathrm{Nu}=\left\{\begin{array}{c}
0.40+0.54 \text { para } 0.1<\operatorname{Re}<1000 \\
0.30 \operatorname{Re}^{0.6} \text { para } 1000<\operatorname{Re}<50000
\end{array}\right.
$$

En las que el número de Reynolds, está dado para una temperatura de aire de $20^{\circ} \mathrm{C}$, calculado por medio de la ecuación 45 :

$\operatorname{Re}=\frac{\rho \cdot \mathrm{V} \cdot \mathrm{D}_{\mathrm{i}}}{\mu}$

Las diferencias entre tener un receptor sin cobertura y un receptor con cobertura se encuentran en el cálculo de la radiación solar total absorbida por el colector (S), y el coeficiente global de pérdidas térmicas, (UL). Los cálculos realizados sirvieron para analizar las variables involucradas en este proyecto.

\section{Descripción del sistema}

El sistema de calentamiento está constituido de 4 superficies parabólicas reflectoras de lámina de acero galvanizada de $0.91 \mathrm{~m}$ de longitud, que tienen como receptores cada una un tubo de cobre de $1 \mathrm{~m}$ y una estructura de ángulos de acero como soporte.

SORIA-MEJÍA, Sandra Paloma, AMBROSIO-JUAREZ, José Emmanuel, TOLEDO-MANUEL, Iván y SABÁS-SEGURA, José Diseño y construcción de un Colector Solar de Tipo Concentrador Parabólico Compuesto (CPC) para pruebas de calentamiento de aire. Revista de Energía Química y Física. 2019 
Este colector solar CPC utiliza la energía solar para calentar el aire circundante en el interior de los tubos, que después puede ser utilizado o almacenado.

El colector debe estar orientado hacia el sur en el hemisferio norte, apuntando hacia el ecuador, y además debe estar colocado a cierta inclinación de manera tal que pueda captar la mayor radiación solar posible.

El funcionamiento es sencillo, por un extremo de los tubos absorbedores entra el aire con una temperatura baja, el cual se va calentando al recorrer la tubería de cobre, y luego sale por otro extremo con una temperatura elevada. Para que el aire circule, no es necesario el uso de bombas o dispositivos similares, funcionando debido al cambio de densidades que el aire sufre al calentarse.

\section{Construcción del Colector CPC}

A continuación, se describe el proceso de construcción del colector solar CPC. Los materiales se describen en la tabla 1 . Primeramente, se cortó la hoja de lámina galvanizada, quedando piezas con dimensiones de $0.375 \times 0.91 \mathrm{~m}$. Para hacer la forma parabólica de los concentradores se doblan cada hoja de lámina mediante una maquina dobladora de accionamiento manual que realiza los pliegues de forma lineal a lo largo de la lámina como se ve en la figura 4.

\begin{tabular}{|c|c|c|}
\hline Cantidad & Material & Dimensiones \\
\hline 1 & $\begin{array}{l}\text { Hoja de lámina de acero } \\
\text { galvanizada }\end{array}$ & $3 \times 0.91 "$ \\
\hline 1 & Tramo de tubo de cobre & $1 "$ \\
\hline 4 & $\begin{array}{l}\text { Tramos de ángulo de } \\
\text { acero }\end{array}$ & $1 \times 1 \times 1 / 8^{6}$ \\
\hline 6 & Tees de cobre & $1 "$ \\
\hline 2 & Codos de cobre & $90^{\circ}, 1^{\prime \prime}$ \\
\hline 20 & Remaches de aluminio & \\
\hline 12 & $\begin{array}{l}\text { Tornillos de cabeza } \\
\text { hexagonal }\end{array}$ & $1 / 4 "$ \\
\hline 12 & Tuercas & $1 / 4 "$ \\
\hline 12 & $\begin{array}{l}\text { Arandelas de presión } \\
\text { Grower }\end{array}$ & $1 / 4 "$ \\
\hline 2 & $\begin{array}{l}\text { Pintura de color gris } y \\
\text { negra }\end{array}$ & \\
\hline
\end{tabular}

Tabla 1 Materiales requeridos para la construcción del colector CPC
A continuación, se realiza la unión entre dos laminas para obtener 4 elementos, por lo cual se soldán en la parte donde se hicieron los pliegues teniendo como resultado los concentradores mostrados en la figura 5 .

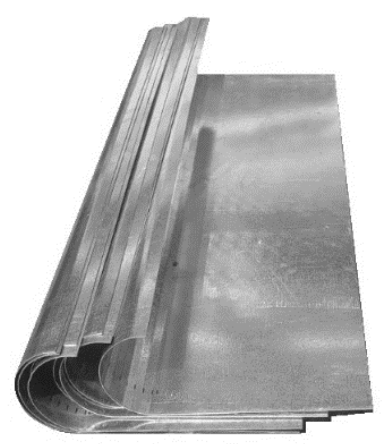

Figura 4 Hojas de lámina galvanizada dobladas

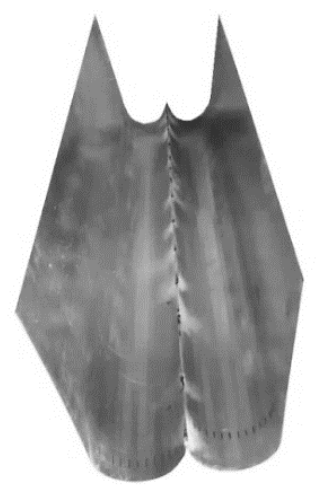

Figura 5 Concentrador ensamblado

Se construyeron recuadros para cada concentrador con los ángulos de acero para soportar estas superficies reflectoras, y estos fueron montados sobre un soporte que sostiene las superficies reflectoras, creando con un ángulo de acero un rectángulo con dimensiones de 1.33 × $0.98 \mathrm{~m}$, la cual esta inclinada a $21^{\circ}$ colocándoles 2 bases y 2 de, figura 6 .

Se cortaron 4 trozos de tubo de cobre de $1 \mathrm{~m}$, para formar un circuito en paralelo usando codos de cobre, fijándose este conjunto después a cada concentrador mediante un soporte hecho, mostrado en la figura 6 . 


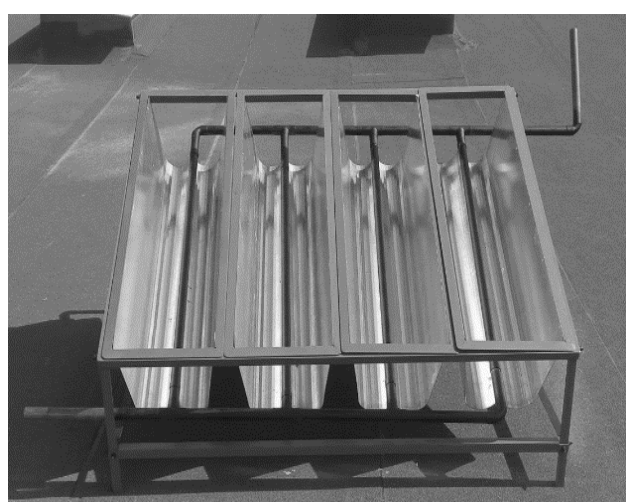

Figura 1 Colector solar tipo CPC

\section{Evaluación Experimental}

Para realizar el análisis térmico del sistema, primeramente, se realizan las mediciones de las variables climatológicas en el lugar de pruebas a cada hora, a lo largo de 7 horas, durante un mes utilizando un termómetro infrarrojo. En la siguiente tabla se muestran los valores promedio obtenidos, en la cual se puede observar que la temperatura de salida en el colector se alcanza a las 3:00 p.m.

\begin{tabular}{|c|c|c|c|c|c|}
\hline Hr. & $\begin{array}{c}\text { Rad. } \\
\text { Solar } \\
(\mathbf{W} / \mathbf{m} \mathbf{2})\end{array}$ & $\begin{array}{c}\text { Temp. } \\
\text { Ambiente } \\
(\mathbf{K})\end{array}$ & $\begin{array}{c}\text { Temp. } \\
\text { Receptor } \\
(\mathbf{K})\end{array}$ & $\begin{array}{c}\text { Temp. } \\
\text { Interior } \\
(\mathbf{K})\end{array}$ & $\begin{array}{c}\text { Temp. } \\
\text { Salida } \\
(\mathbf{K})\end{array}$ \\
\hline 11:00 & 640.60 & 294.75 & 303.55 & 301.55 & 306.95 \\
\hline $12: 00$ & 814.80 & 296.45 & 312.55 & 305.15 & 318.85 \\
\hline $13: 00$ & 803.60 & 297.15 & 320.05 & 309.25 & 323.35 \\
\hline $14: 00$ & 792.90 & 299.45 & 323.95 & 310.05 & 326.85 \\
\hline $15: 00$ & 530.00 & 300.05 & 330.95 & 311.75 & 334.25 \\
\hline $16: 00$ & 525.60 & 299.55 & 310.25 & 306.05 & 319.75 \\
\hline $17: 00$ & 101.70 & 293.55 & 289.25 & 295.45 & 298.55 \\
\hline
\end{tabular}

Tabla 2 Mediciones en el CPC

En el gráfico 1, se puede apreciar que la temperatura de salida del colector tiende a seguir el comportamiento de la temperatura ambiente, aumentado considerablemente e influyendo de forma notable en el rendimiento del sistema.

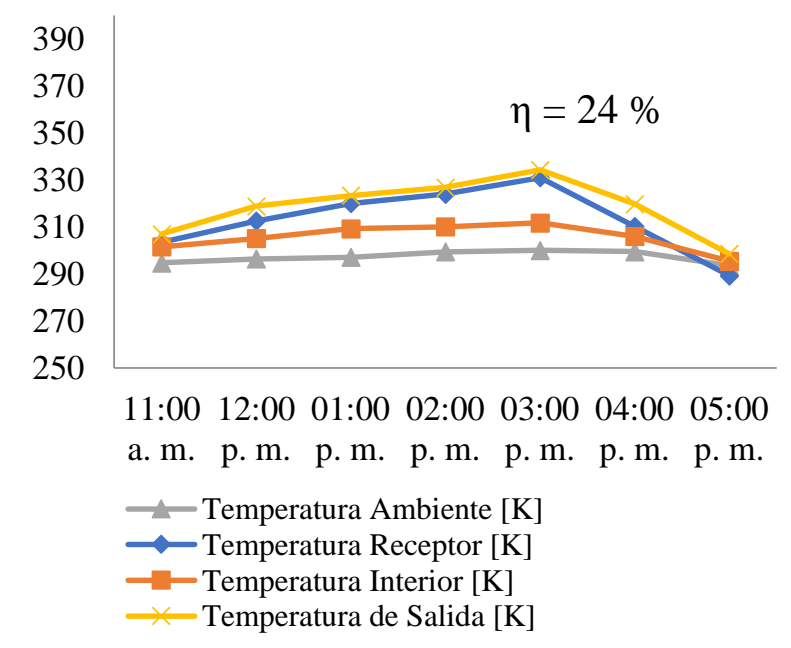

Gráfico 1 Gráfica de las temperaturas en el CPC.
Para conocer la cantidad de radiación solar absorbida por el colector CPC, se hallan los valores necesarios para cada día de pruebas, obteniendo como promedios los expresados en la tabla 3 , donde se toman en cuenta los valores geométricos del CPC, los ángulos del movimiento aparente del sol y los datos geográficos del lugar donde se realizaron las pruebas.

Con los parámetros de diseño efectuados en el capítulo 2, se presentan a continuación en la tabla 4 las características de cada concentrador, las cuales son basadas básicamente en el diámetro del receptor y el ángulo de aceptación propuesto.

\begin{tabular}{|c|c|c|c|}
\hline & Símbolo & Valor & Unidad \\
\hline $\begin{array}{l}\text { Irradiancia } \\
\text { promedio }\end{array}$ & IT & 5900 & $\mathrm{Wh} / \mathrm{m} 2$ \\
\hline Horas Solar Pico & HSP & 7 & $\mathrm{~h}$ \\
\hline \begin{tabular}{|l}
$\begin{array}{l}\text { Radiación solar } \\
\text { total }\end{array}$ \\
\end{tabular} & G & 790.0119 & $\mathrm{~W} / \mathrm{m} 2$ \\
\hline Radiación directa & $\mathrm{Gb}$ & 602.4938 & $\mathrm{~W} / \mathrm{m} 2$ \\
\hline Radiación difusa & Gd & 187.5181 & $\mathrm{~W} / \mathrm{m} 2$ \\
\hline $\begin{array}{l}\text { Radiación } \\
\text { extraterrestre }\end{array}$ & Go & 1345.5766 & $\mathrm{~W} / \mathrm{m} 2$ \\
\hline Constante solar & Gsc & 1367 & $\mathrm{~W} / \mathrm{m} 2$ \\
\hline $\begin{array}{l}\text { Transmitancia } \\
\text { atmosférica para la } \\
\text { radiación directa }\end{array}$ & $\tau_{\mathrm{b}}$ & 0.4478 & \\
\hline $\begin{array}{l}\text { Transmitancia } \\
\text { atmosférica para la } \\
\text { radiación difusa }\end{array}$ & $\tau_{\mathrm{d}}$ & 0.1394 & \\
\hline \multirow{3}{*}{$\begin{array}{l}\text { Valores para } \\
\text { atmósfera estándar }\end{array}$} & $\mathrm{a} 0$ & 0.2729 & \\
\hline & a1 & 0.3690 & \\
\hline & $\mathrm{K}$ & 0.2595 & \\
\hline Altitud del lugar & A & 1.7110 & $\mathrm{~km}$ \\
\hline Ángulo cenital & $\cos \theta z$ & 0.3476 & $\mathrm{o}$ \\
\hline Latitud del lugar & $\Phi$ & 20.4399 & o \\
\hline Declinación solar & $\Delta$ & 14.5856 & o \\
\hline Inclinación & B & 21 & o \\
\hline Ángulo acimutal & $\gamma$ & -101.5457 & o \\
\hline Ángulo horario & $\Omega$ & 73.3624 & o \\
\hline Tiempo solar & Tsun & 16.8908 & \\
\hline Tiempo estandar & Tclock & 15 & \\
\hline \multirow{2}{*}{\begin{tabular}{|ll} 
Ecuación del \\
tiempo
\end{tabular}} & ET & 0.0908 & \\
\hline & B & 38.4658 & \\
\hline Longitud estandar & Lstd & -102 & o \\
\hline Longitud local & Lloc & -101.55 & o \\
\hline $\begin{array}{l}\text { Constante de Stefan } \\
\text { Boltzmann }\end{array}$ & $\Sigma$ & $5.67 \times 10-8$ & $\mathrm{~W} / \mathrm{m} 2 \mathrm{~K} 4$ \\
\hline
\end{tabular}

Tabla 3 Datos considerados para los cálculos de radiación solar

La superficie receptora como se ha mencionado anteriormente es un tubo de cobre, que por su durabilidad, su propiedad anticorrosiva y sus propiedades térmicas, ha sido elegido para este proyecto. 
En la tabla 5, se muestran las propiedades que se tomaron en cuenta para los cálculos, considerando que la superficie fue pintada de color negro para una mayor absorbancia de calor.

\begin{tabular}{|l|l|r|l|}
\hline & Símbolo & Valor & \multicolumn{1}{|c|}{ Unidad } \\
\hline Semiángulo de aceptación & $\mathrm{Ga}$ & 23.5 & ${ }^{\circ}$ \\
\hline Concentración geométrica & $\mathrm{C}$ & 2.5 & \\
\hline Longitud de abertura & $\mathrm{La}$ & 0.23 & $\mathrm{~m}$ \\
\hline Altura del CPC & $\mathrm{H}$ & 0.32 & $\mathrm{~m}$ \\
\hline Longitud del CPC & $\mathrm{L}$ & 0.91 & $\mathrm{~m}$ \\
\hline Diámetro exterior del tubo & $\mathrm{De}$ & 0.028575 & $\mathrm{~m}$ \\
\hline Diámetro interior del tubo & $\mathrm{Di}$ & 0.0254 & $\mathrm{~m}$ \\
\hline Espesor del receptor & $\mathrm{E}$ & 0.003175 & $\mathrm{M}$ \\
\hline Área de abertura & $\mathrm{Aa}$ & 0.2093 & $\mathrm{~m} 2$ \\
\hline Área del receptor & $\mathrm{Ar}$ & 0.0817 & $\mathrm{~m} 2$ \\
\hline $\begin{array}{l}\text { Número total } \\
\text { concentradores }\end{array}$ & & 4 & \\
\hline
\end{tabular}

Tabla 4 Características de cada CPC del colector solar

\begin{tabular}{|c|c|c|c|}
\hline & Símbolo & Valor & Unidad \\
\hline Conductividad térmica & $\mathrm{Kr}$ & 385000 & $\mathrm{~W} / \mathrm{m}^{\circ} \mathrm{C}$ \\
\hline Absortividad & $\alpha r$ & 1 & \\
\hline Emisividad & $\varepsilon \mathrm{r}$ & 0.037 & \\
\hline Transmitancia & $\tau_{\mathrm{r}}$ & 1 & \\
\hline
\end{tabular}

Tabla 5 Propiedades del receptor.

La superficie reflectora elegida es la lámina de acero galvanizada que es cortada en segmentos y de la cual se requiere conocer para el cálculo térmico la reflectividad especular de esta, mostrándose en la tabla 6.

\begin{tabular}{|l|l|l|l|}
\cline { 2 - 4 } \multicolumn{1}{c|}{} & Símbolo & Valor & Unidad \\
\hline Reflectividad especular & $\rho \mathrm{r}$ & 0.78 & \\
\hline
\end{tabular}

Tabla 6 Reflectividad especular de la lámina galvanizada.

El fluido de trabajo en este proyecto es el aire, pensando en las aplicaciones futuras que se pudieran considerar, donde en la tabla 7 , se muestran las propiedades de este a $20^{\circ} \mathrm{C}$, así como también la velocidad promedio que se presenta en los días de pruebas.

\begin{tabular}{|l|l|r|l|}
\cline { 2 - 4 } \multicolumn{1}{c|}{} & Símbolo & \multicolumn{1}{l}{ Valor } & Unidad \\
\hline Conductividad térmica & Kaire & 0.02476 & $\mathrm{~W} / \mathrm{m} 2 \mathrm{~K}$ \\
\hline Calor especifico & $\mathrm{Cp}$ & 1007 & $\mathrm{~J} / \mathrm{kgK}$ \\
\hline Densidad & $\mathrm{P}$ & 1.204 & $\mathrm{~kg} / \mathrm{m} 3$ \\
\hline Velocidad & $\mathrm{V}$ & 0.1636 & $\mathrm{~m} / \mathrm{s}$ \\
\hline Viscosidad dinámica & $\mathrm{M}$ & $1.825 \times 10-5$ & $\mathrm{~kg} / \mathrm{ms}$ \\
\hline
\end{tabular}

Tabla 7 Propiedades del aire a $20^{\circ} \mathrm{C}$.

Conociendo las propiedades y los datos geométricos de los elementos que conforman el colector CPC, se procede a hallar las pérdidas térmicas y la eficiencia del colector CPC, en donde la tabla 8 muestra los resultados obtenidos.

\begin{tabular}{|c|c|c|c|}
\hline & Símbolo & Valor & Unidad \\
\hline Eficiencia del sistema & $\mathrm{H}$ & 23 & $\%$ \\
\hline Área del colector & $\mathrm{Ac}$ & 0.8372 & $\mathrm{~m} 2$ \\
\hline Calor útil & $\mathrm{Qu}$ & 95.0274 & $\mathrm{~W}$ \\
\hline Radiación solar absorbida & $\mathrm{S}$ & 495.7459 & $\mathrm{~W} / \mathrm{m} 2$ \\
\hline Transmitancia del CPC & $\tau_{\mathrm{CPC}}$ & 0.7495 & \\
\hline $\begin{array}{l}\text { Número promedio de } \\
\text { reflexiones }\end{array}$ & $\mathrm{nr}$ & 0.9574 & \\
\hline $\begin{array}{l}\text { Coeficiente global de } \\
\text { pérdidas de calor }\end{array}$ & $\mathrm{UL}$ & 0.6816 & $\mathrm{~W} / \mathrm{m} 2 \mathrm{~K}$ \\
\hline $\begin{array}{l}\text { Perdida térmica del } \\
\text { receptor }\end{array}$ & Qloss & 0.1206 & W \\
\hline $\begin{array}{l}\begin{array}{l}\text { Factor de flujo } \\
\text { colector }\end{array}\end{array}$ & $F^{\prime \prime}$ & 0.9984 & \\
\hline $\begin{array}{l}\text { Factor de eliminación de } \\
\text { calor del colector }\end{array}$ & FR & 0.9282 & \\
\hline $\begin{array}{l}\text { Factor de eficiencia del } \\
\text { colector }\end{array}$ & $F^{\prime}$ & 0.9297 & \\
\hline $\begin{array}{l}\text { Coeficiente global de } \\
\text { transferencia de calor }\end{array}$ & Uo & 0.6337 & $\mathrm{~W} / \mathrm{m} 2 \mathrm{~K}$ \\
\hline Flujo másico del aire & $\dot{m}$ & 0.0161 & $\mathrm{~kg} / \mathrm{s}$ \\
\hline $\begin{array}{l}\text { Coeficiente de } \\
\text { transferencia de calor } \\
\text { dentro del tubo receptor }\end{array}$ & hfi & 10.1406 & $\mathrm{~W} / \mathrm{m} 2 \mathrm{~K}$ \\
\hline Número de Nusselt & Un & 10.4027 & \\
\hline Número de Reynolds & $\operatorname{Re}$ & 274.1121 & \\
\hline
\end{tabular}

Tabla 8 Características geométricas y parámetros de cálculo en el colector solar tipo CPC

Se tiene que para estos días de pruebas se consigue una eficiencia del $23 \%$ en promedio, siendo este factor importante en el diseño de colectores, ya que significa tener una ganancia al retornar de forma útil sólo una parte de la energía que recibe.

\section{Conclusiones}

Al ser un colector de tipo estacionario se consigue una baja concentración, obteniendo una radiación solar en el colector aceptable. La factibilidad de este trabajo es que cuenta con concentradores como equipos principales hace que se considere al colector cilindro parabólico compuesto como un camino viable tanto económicamente como energéticamente para sistemas que requieran mantener una temperatura baja en horas solares punta.

Mediante los resultados obtenidos en este proyecto se muestra que los resultados teóricos y experimentales son similares, así mismo el diseño probado permite que solo parte de la radiación entre al colector permitiendo obtener temperaturas de entre 50 y $60{ }^{\circ} \mathrm{C}$. La eficiencia alcanzada por este proyecto es del $24 \%$, con temperaturas de salida de $60{ }^{\circ} \mathrm{C}$, lo cual resulta adecuado para ser implementado en sistemas que requieran calor constante a temperaturas relativamente bajas, tales como invernaderos o sistemas de climatización. 


\section{Referencias}

Altaif, R. d. (Mayo de 2015). Estudio numérico experimental del distribuidor hidráulico de un colector solar de tubo al vacío tipo heat-pipe. Obtenido de

http://eprints.uanl.mx/9538/1/1080214942.pdf

Aschwanden, M. J. (2014). Physics of the Solar Corona. An Introduction. Praxis Publishing.

ASHRAE. (2011).

Bahng, J., \& Schwarzchild, M. (1961). Lifetime os Solar Granules. The Astrophysical Journal.

Barreto, J. A. (Julio de 2008).

Castells, X. E. (2012). Energías Renovables. Madrid: Ediciones Díaz de Santos Albasanz.

Chaves, I. M. (junio de 2015). DISEÑO DE COCINAS. Obtenido de DISEÑO DE COCINAS.

Duffie, J., \& Beckman, W. (2013). Solar Engineering of Thermal Processes. Canadá: John Wiley \& Sons, Inc.

Elortegui, N., \& Jarabo, F. (2000). Energías Renovables. Madrid: SAPT Publicaciones Técnicas.

Española, R. A. (octubre de 2014). Diccionario de la lengua española. Madrid.

Falcón, N., Peña, F., Mavo, H., \& Muñoz, R. (2001). Irradiación solar global en la ciudad de Valencia. INGENIERÍA UC [en linea] 2001, 8 (diciembre).

Lovegrove, K., \& Stein, W. (2012). Concentrating Solar Power Technology: Principles, Developments and Applications. Australia: Woodhead Publishing.

Martínez, M. P. (2017). La hora solar pico equivalente. Definición e interpretación. Scielo, Ingeniería Energética vol.38 no.2. Versión en línea ISSN 1815-5901.

Martínez, P. R. (2010). Energia Solar Térmica: Técnicas para su Aprovechamiento. Barcelona: Marcombo Ediciones Técnicas.

Viloria, J. R. (2011). Estudios de viabilidad de instalaciones solares. Determinación del potencial solar. España: Paraninfo. 